\title{
RELACIONES PÚBLICAS. Estrategias implementadas por el Instituto para la exportación y la moda - INEXMODA
}

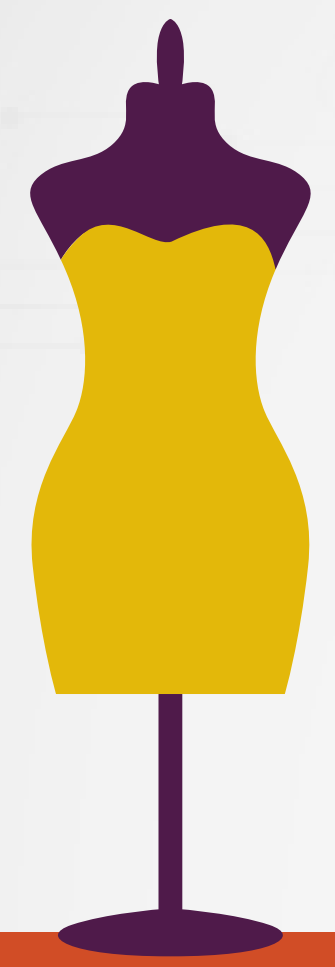

\section{RESUMEN}

El artículo presenta las estrategias de relaciones públicas utilizadas en el Instituto para la Exportación y la Moda (INEXMODA), que han contribuido a la consolidación de este sector en Colombia y Latinoamérica; se destaca la realización de ferias, la utilización del benchmarking así como del capital social, las alianzas estratégicas y la utilización de legitimadores en sus acciones.

Palabras claves: Relaciones Públicas, capital social, comunicación estratégica 


\section{PUBLIC RELATIONS. Strategies implemented by the Institute for export and fashion - INEXMODA}

\section{SUMMARY}

This article presents the public relations strategies implemented by the Institute for Export and Fashion (INEXMODA), which strengthened the role of Medellin as a fashion cluster in Colombia and Latin America; It stands out the realization of fairs, the use of benchmarking as well as social capital and corporate philosophy in each of its actions.

Keywords: Publicity, social capital, strategic communication

\section{RELAÇÕES PÚBLICAS}

\section{Estratégias implementadas pelo Instituto para a exportação e a moda - INEXMODA}

\section{RESUMO}

O artigo apresenta as estratégias de relações públicas usadas no Instituto para a Exportação e a Moda (INEXMODA), que contribuíram à consolidação de Medellín como cidade da moda na Colômbia e América

Latina. Destaca-se a realização de feiras, o uso de benchmarking, bem como capital social e filosofia corporativa em cada uma de suas ações.

Palavras chaves: Relações Públicas, capital social, comunicação estratégica. 


\section{INTRODUCCIÓN}

$E^{\prime}$ I Instituto para la exportación y moda INEXMODA, fue fundado en 1988 en MedellínColombia, por Roque Ospina. Alicia Mejía [1] fue su primera Directora de Mercadeo y Clara Echeverri, directora de negocios internacionales. Desde 1985, la Asociación Nacional de Industriales (ANDI), se planteó la necesidad de un instituto de este tipo, con el propósito de que se encargara de coordinar los intangibles de la moda, tales como la gestión de las Relaciones Públicas de este sector.

Este Instituto, creó la semana de la moda, así como las dos ferias comerciales más conocidas del País: COLOMBIATEX y COLOMBIAMODA, eventos en los que las Relaciones Públicas, han sido fundamentales; como por ejemplo, cuando se logró que el diseñador Oscar de la Renta, participara en estas, en 1999, momento en el que la ciudad contaba con una imagen negativa debido a la violencia y el narcotráfico.

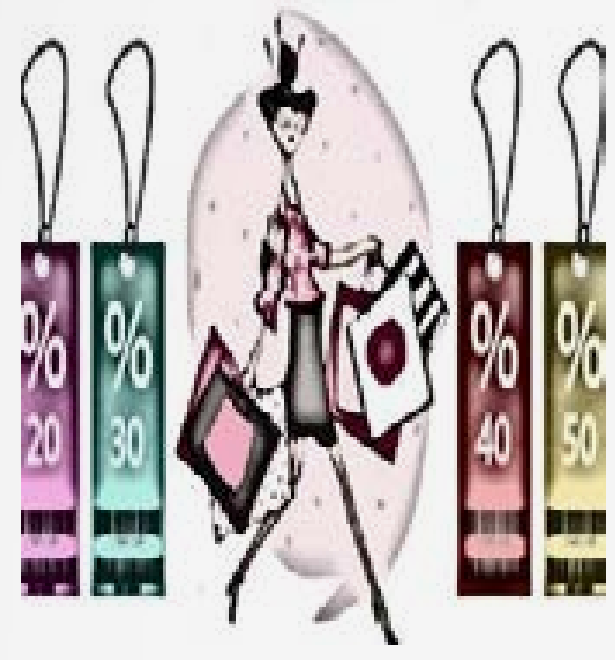

Diversos análisis se han realizado en torno a este tema, Agudelo (2015) realizó un estudio de caso acerca de INEXMODA, en el que abordó Las ferias como escenarios de comunicación y promoción de la ciudad, en este indagó desde la perspectiva funcionalista, la influencia de las acciones de comunicación realizadas por el Instituto en COLOMBIAMODA, en el año 2013, y examinó el rol de las estrategias de la feria en la promoción de Medellín ante nacionales y extranjeros. Por su parte, Gartner en el (2012) reflexionó sobre El vestir como referente de transformaciones sociales en Medellín: un análisis de la industrialización textil y del surgimiento de INEXMODA como estrategias de modernización y cambio social; con mirada histórico-sociológica, muestra la influencia que tuvo el surgimiento de dicho instituto, en la estética mercantil que referencia la transformación social de este lugar.

En este artículo se describe la evolución de las estrategias de relaciones públicas en INEXMODA; y se estima el impacto de estas en el posicionamiento de Medellín como ciudad de moda.

\section{Metodología}

En este caso de estudio se utilizó metodología cualitativa. Tuvo por objetivo analizar las estrategias de relaciones públicas en INEXMODA. Para Bautu (2012), la metodología cualitativa se apoya en:

"la idea de la unidad de la realidad holística y en la fidelidad a la perspectiva de los actores involucrados en esa realidad" (p.32).
El estudio de caso se entiende como "un método de investigación centrado en el abordaje holístico de un fenómeno, dentro de su contexto real, en el que los límites entre el fenómeno y su contexto requiere múltiples fuentes de información" (Martínez, 1994: 17). Los agentes sociales, informantes, fueron punto central en esta investigación, "sus percepciones, ideas, emociones e interpretaciones, constituyen la investigación misma" (Bautú, p. 38). Los entrevistados clave fueron María Alicia Mejía (exdirectora de mercadeo), Julián Posada (ex asesor, durante y después del período de Mejía) y Martha Cálad (directora del laboratorio de moda).

Para el estudio se seleccionaron tres momentos de INEXMODA: el primero, 1989 y 1990: comienzo de la gestión de María Alicia Mejía; el segundo, entre los años 1999 y 2005; y el tercero, el Instituto en la actualidad. Se revisaron distintas publicaciones impresas y la web. Se realizó revisión documental en los periódicos El Colombiano, El Tiempo, EI Mundo, EI Espectador y en la revista Semana, entre otros medios. Para la sistematización se utilizó el software Atlas. Ti y Excel. Los acontecimientos que se encontraron en las publicaciones se ordenaron según las siguientes categorías (ver tabla N1).

- Entidad Internacional (publicaciones centradas en un personaje u organización extranjeros que validan el acontecimiento)

- Cifras (publicaciones que se centran en mediciones y asuntos económicos)

- Datos (publicaciones con información fuera de los asuntos económicos)

- Noticia (publicación que se presenta como un hecho noticioso)

- Reseña (publicaciones que hablan de asuntos biográficos o aspectos generales). 
Tabla N1.

Sistematización de Publicaciones sobre INEXMODA

\begin{tabular}{|c|c|c|c|c|c|}
\hline $\begin{array}{l}\text { Nombre del } \\
\text { medio }\end{array}$ & $\begin{array}{l}\text { Fecha de } \\
\text { publicación }\end{array}$ & $\begin{array}{l}\text { Alcance del } \\
\text { medio }\end{array}$ & Tipo de medio & Tipo de acontecimiento & Contenido de la publicación \\
\hline Semana & $26 / 01 / 2004$ & Nacional & Revista & Entidad internacional & $\begin{array}{c}\text { Exposición alta costura de Balmain en } \\
\text { Medellín }\end{array}$ \\
\hline La W & $14 / 07 / 2003$ & Nacional & Radio & Entidad internacional & Mario Bocelli en Medellín \\
\hline El Tiempo & $4 / 11 / 2003$ & Nacional & Periódico & Entidad internacional & $\begin{array}{c}\text { Exposición alta costura de Balmain en } \\
\text { Bogotá }\end{array}$ \\
\hline Fucsia & $3 / 09 / 2000$ & Nacional & Revista & Entidad internacional & $\begin{array}{l}\text { Periodista Lola Gavarrón en Colom- } \\
\text { biamoda }\end{array}$ \\
\hline Colarte & $? / 08 / 2002$ & Nacional & Portal web & Entidad internacional & Ágatha Ruíz de la Prada en Medellín \\
\hline Colarte & $? / 08 / 2002$ & Nacional & Portal web & Entidad internacional & Óscar de la Renta en Medellín \\
\hline Colarte & $? / 08 / 2002$ & Nacional & Portal web & Entidad internacional & Armando Mafud en Medellín \\
\hline Colarte & $? / 08 / 2002$ & Nacional & Portal web & Entidad internacional & Badgley \& Mischka en Medellín \\
\hline Extroversia & $08 / 10 / 2005$ & Nacional & Portal web & Reseña & Identidad Colombia en Milán \\
\hline Semana & $8 / 07 / 2006$ & Nacional & Revista & Cifras & Desarrollo y crecimiento de las ferias \\
\hline El Colombiano & 29/07/200? & Regional & Periódico & Datos & $\begin{array}{l}\text { Contexto de Medellín al comienzo de } \\
\text { las ferias }\end{array}$ \\
\hline Semana & $11 / 09 / 2000$ & Nacional & Revista & Entidad Internacional & Desfile de Balmain en Medellín \\
\hline Semana & $11 / 09 / 2000$ & Nacional & Revista & Reseña & Crecimiento de Inexmoda \\
\hline Fucsia & $3 / 09 / 2000$ & Nacional & Revista & Entidad internacional & Lola Gavarrón en Medellín \\
\hline Fucsia & $3 / 09 / 2000$ & Nacional & Revista & Entidad internacional & Alain Hivelin en Medellín \\
\hline Fucsia & $10 / 04 / 2004$ & Nacional & Revista & Reseña & Identidad Colombia en Milán \\
\hline Jet-Set & $13 / 08 / 2015$ & Nacional & Revista & Reseña & Alessandra Ambrosio en Medellín \\
\hline Presidencia & $30 / 09 / 2004$ & Nacional & Boletín & Noticia & Identidad Colombia en Milán \\
\hline El Tiempo & $21 / 01 / 2004$ & Nacional & Periódico & Noticia & Exposición Balmain en Medellín \\
\hline El Tiempo & $24 / 07 / 2010$ & Nacional & Periódico & Reseña & $\begin{array}{c}\text { Esteban Cortazar de Ungaro regresa } \\
\text { a Medellin }\end{array}$ \\
\hline EI Tiempo & $25 / 01 / 2004$ & Nacional & Periódico & Noticia & Identidad Colombia en Milán \\
\hline El Tiempo & $23 / 07 / 2015$ & Nacional & Periódico & Reseña & Haider Ackermann en Medellín \\
\hline El Mundo & $03 / 07 / 2008$ & Local & Periódico & Reseña & Convenio Avianca - Inexmoda \\
\hline El Mundo & $26 / 07 / 2009$ & Local & Periódico & Noticia & Carolina Herrera en Medellín \\
\hline El Mundo & $26 / 07 / 2009$ & Local & Periódico & Noticia & Clara Echeverri sale de Inexmoda \\
\hline El Mundo & $28 / 07 / 2009$ & Local & Periódico & Datos & $\begin{array}{l}\text { Prensa internacional en Colombia- } \\
\text { moda }\end{array}$ \\
\hline El Mundo & $28 / 07 / 2009$ & Local & Periódico & Entidad internacional & Carolina Herrera en Medellín \\
\hline El Mundo & $28 / 07 / 2009$ & Local & Periódico & Entidad internacional & Loewe en Medellín \\
\hline El Mundo & $28 / 07 / 2009$ & Local & Periódico & Entidad Internacional & Custo Barcelona en Medellín \\
\hline Semana & $11 / 09 / 2000$ & Nacional & Revista & Entidad internacional & Agatha Ruíz de la Prada en Medellín \\
\hline $\begin{array}{l}\text { La Hoja de } \\
\text { Medellín }\end{array}$ & jul-96 & Local & Revista & Reseña & Contexto de Alicia Mejía \\
\hline El Colombiano & $29 / 05 / 1989$ & Regional & Periódico & Reseña & $\begin{array}{c}\text { Colombia se prepara como centro } \\
\text { textil }\end{array}$ \\
\hline El Colombiano & $29 / 05 / 1989$ & Regional & Periódico & Cifras & Exportaciones de textiles \\
\hline El Colombiano & $30 / 05 / 1989$ & Regional & Periódico & Cifras & Exportaciones de textiles \\
\hline El Colombiano & $30 / 05 / 1989$ & Regional & Periódico & Reseña & Clara Echeverri de Inexmoda \\
\hline El Colombiano & $30 / 05 / 1989$ & Regional & Periódico & Reseña & Roque Ospina de Inexmoda \\
\hline El Colombiano & $30 / 05 / 1989$ & Regional & Periódico & Reseña & Alicia Mejía de Inexmoda \\
\hline El Colombiano & $30 / 05 / 1989$ & Regional & Periódico & Reseña & Inexmoda como Instituto \\
\hline El Colombiano & $30 / 05 / 1989$ & Regional & Periódico & Reseña & $\begin{array}{l}\text { Nota periodística de la primera Co- } \\
\text { lombiatex }\end{array}$ \\
\hline El Colombiano & $30 / 05 / 1989$ & Regional & Periódico & Entidad Internacional & Ramón Corbera en Medellín \\
\hline El Colombiano & $30 / 05 / 1989$ & Regional & Periódico & Entidad Internacional & Misión española en Colombiatex \\
\hline El Colombiano & $30 / 05 / 1989$ & Regional & Periódico & Reseña & Coltejer en Colombiamoda \\
\hline El Colombiano & 20/08/1999 & Regional & Periódico & Entidad Internacional & Óscar de la Renta en Medellín \\
\hline El Colombiano & $24 / 08 / 1999$ & Regional & Periódico & Entidad Internacional & Óscar de la Renta en Medellín \\
\hline El Colombiano & $24 / 08 / 1999$ & Regional & Periódico & Reseña & Lo que se verá en Colombiamoda 99 \\
\hline El Colombiano & $24 / 08 / 1999$ & Regional & Periódico & Reseña & Cubrimiento de la feria online \\
\hline El Colombiano & 24/08/1999 & Regional & Periódico & Reseña/cifras & Roque Ospina y exportaciones \\
\hline
\end{tabular}




\begin{tabular}{|c|c|c|c|c|c|}
\hline El Colombiano & 25/08/1999 & Regional & Periódico & Noticia & Desfile de Warner Bross en Medellín \\
\hline El Colombiano & 25/08/1999 & Regional & Periódico & Noticia & Desfile de Éxito en Medellín \\
\hline El Colombiano & 25/08/1999 & Regional & Periódico & Entidad Internacional & Elio Firucci en Medellín \\
\hline El Colombiano & 25/08/1999 & Regional & Periódico & Reseña & $\begin{array}{c}50 \text { años del Éxito en la moda y su } \\
\text { influencia }\end{array}$ \\
\hline El Colombiano & 25/08/1999 & Regional & Periódico & Reseña & Recorrido por la feria \\
\hline El Colombiano & 25/08/1999 & Regional & Periódico & Noticia & $\begin{array}{c}\text { Concurso internacional Elite Model } \\
\text { Look }\end{array}$ \\
\hline El Colombiano & 26/08/1999 & Regional & Periódico & Entidad internacional & Óscar de la Renta en Medellín \\
\hline El Colombiano & 26/08/1999 & Regional & Periódico & Cifras & Nuevos aires en el negocio textil \\
\hline El Colombiano & 26/08/1999 & Regional & Periódico & Entidad internacional & Medellín se viste de moda \\
\hline El Colombiano & 26/08/1999 & Regional & Periódico & Reseña & $\begin{array}{c}12 \text { diseñadores colombianos en la } \\
\text { feria }\end{array}$ \\
\hline El Colombiano & 26/08/1999 & Regional & Periódico & Cifras & Reactivación del sector textil \\
\hline El Colombiano & 27/08/1999 & Regional & Periódico & Reseña & Desfile de Alonso Uribe por la paz \\
\hline El Colombiano & 27/08/1999 & Regional & Periódico & Reseña & Pasarela Americamoda Orbitel \\
\hline El Colombiano & 27/08/1999 & Regional & Periódico & Cifras & $\begin{array}{c}\text { Datos económicos Pymes del sector } \\
\text { textil }\end{array}$ \\
\hline El Colombiano & 27/08/1999 & Regional & Periódico & Entidad internacional & Óscar de la Renta en Medellín \\
\hline El Tiempo & 24/08/1999 & Nacional & Periódico & Noticia & Comienzo de Colombiamoda99 \\
\hline El Tiempo & 25/08/1999 & Nacional & Periódico & Noticia & $\begin{array}{l}\text { Problemas tributarios para el sector } \\
\text { textil }\end{array}$ \\
\hline El Tiempo & 26/08/1999 & Nacional & Periódico & Entidad internacional & Óscar de la Renta en Medellín \\
\hline El Tiempo & 26/08/1999 & Nacional & Periódico & Reseña & Reseña general de Colombiamoda99 \\
\hline El Tiempo & 26/08/1999 & Nacional & Periódico & Entidad internacional & Elio Firucci en Medellín \\
\hline El Espectador & 25/08/1999 & Nacional & Periódico & Reseña & Medellín, la gran pasarela \\
\hline El Espectador & $25 / 08 / 1999$ & Nacional & Periódico & Reseña & Desfile de Leonisa en Colombiamoda \\
\hline El Espectador & 25/08/1999 & Nacional & Periódico & Reseña & Desile "puntadas por la paz" \\
\hline
\end{tabular}

El publicity, se midió con fundamento en el concepto de equivalencia publicitaria, en el que se señala la diferencia entre el hecho noticioso y la pauta, también se tuvo en cuenta, la rentabilidad de la aparición de INEXMODA en medios de comunicación.

2. Evolución de las estrategias de Relaciones Públicas en la gestión de la comunicación en INEXMODA: referentes europeos, capital social, apoyo interinstitucional y validadores del sector

\subsection{Benchmarking y referentes europeos}

En 1988, cuando se asignó a Alicia Mejía como directora de mercadeo, la junta Directiva de la ANDI, le encomendó visitar Europa, el propósito, aprender a hacer Ferias. Al respecto comenta Mejía:

"Gilberto Echeverri, Abel Pérez Gil y Pacho o Carlos Alberto Roble, me dijeron, ¿por qué no te vas para Barcelona, un mecesito, para que veas como se hace la feria Fira? nosotros tenemos allá unos contactos. En ese momento, esa feria era importantísima, Barcelona fue una ciudad textil muy destacada. Ya no es así, España ya no tiene esa fortaleza... 


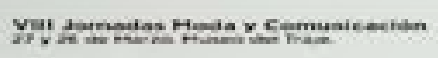

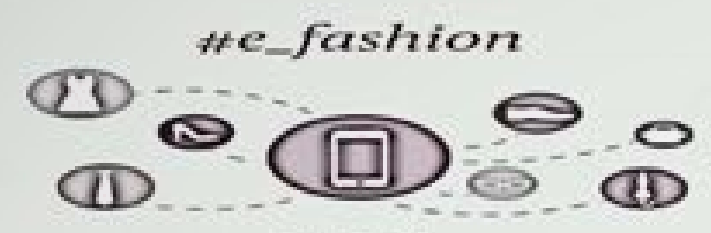

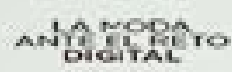

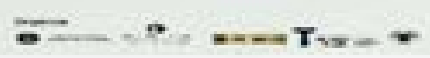

Lo que aprendimos en Premiere Vision y demás ferias internacionales, se ha aplicado en nuestras ferias nacionales... indudablemente, han sido punto de referencia, pero de todas maneras hay que hacer una adaptación de acuerdo a las necesidades de los industriales del país, de nuestros visitantes y compradores, de nuestra cultura, idiosincrasia. Entonces, obviamente aprendimos mucho, de las ferias internacionales, pero las ferias que tenemos hoy, es el resultado de entender nuestro entorno, porque nosotros no estamos trabajando para europeos, sino para colombianos y latinoamericanos, principalmente...

Martha Cálad, 2016

Directora del laboratorio de moda

Bueno, yo aterricé en Barcelona. Fira es inmenso. Yo me reunía con el encargado de mercadeo, con el de comunicaciones, con el de relaciones públicas, con el que hacía la cocina, con el que montaba, con el de los paneles, el de los tapetes, el de las fotografías, el de las luces. $Y$ preguntaba, preguntaba ¿Cómo es una rueda de prensa? ¿Me dejan tomar una foto? Me acuerdo que mantenía unas tijeritas, y entonces veía un tapete que me gustaba y preguntaba ¿Me dejan cortar un pedacito? Nosotros [los antioqueños] no somos bobos. Allá la gente me recibió con la más absoluta generosidad.

Alicia Mejía, 2016.

Con esas pasantías a Europa, Mejía, conoció el proceso y desarrollo de las ferias: Premiere Vision de Francia y Fira en España, lo aprendido lo adaptó a las necesidades de INEXMODA en Colombia. Para Wilcox (2006), las Relaciones Públicas pueden contribuir a alcanzar objetivos de marketing, y la disciplina es asumida como un "concepto de planificación global que evalúa el papel estratégico de una diversidad de disciplinas de comunicación, para lograr un impacto claro, consistente y máximo de la comunicación" ( $p$. 20).

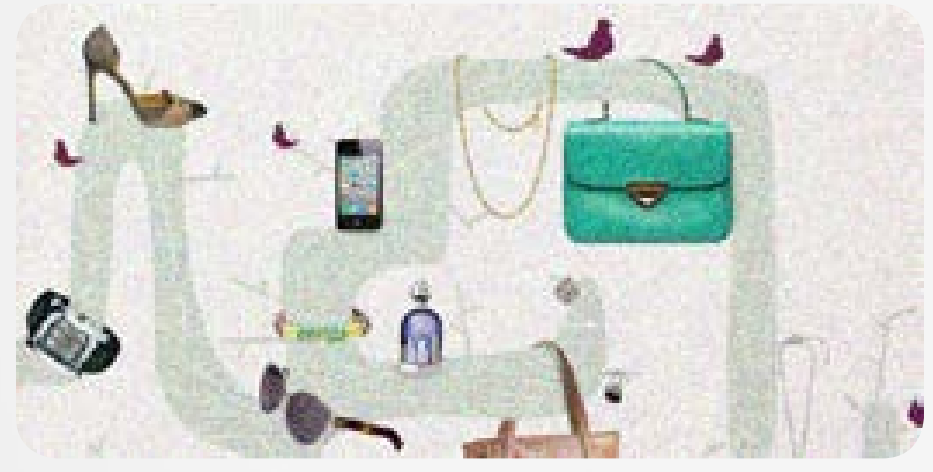

Julián Posada, quien trabajó conjuntamente con Alicia Mejía, comenta que en ese primer periodo de INEXMODA se hicieron estudios de benchmarking, con el fin de analizar el público. Estos fueron implementados en distintas áreas del Instituto.

Nosotros nos manteníamos mucho tiempo del año en aviones, buscando gente que nos pudiera venir a alimentar, a nutrir, a enseñar, a indicar, a capacitar, a aprender, a ver. Contactábamos amigas que estarían en los desfiles; por ejemplo recuerdo que en un año "una amiga en Francia, nos envió toda la información de los desfiles". En INEXMODA decíamos "tenemos que conocer la sala de prensa del New York Fashion Week", ¿me entiendes? Y allá nos íbamos. Es que hay que aprender de los que están haciendo las cosas bien. ¡Teníamos mentalidades realmente muy amplias!

Julián Posada, 2016.

Desde la experiencia de INEXMODA, los referentes internacionales enseñan, pero ante todo hay que adaptar el conocimiento a las circunstancias de cada feria.

\subsection{Capital social como potenciador de las estrategias de Relaciones Públicas}

Bourdieu, en Poder, derecho y clases sociales (1983), define el capital social como una "constitución de recursos potenciales $\mathrm{o}$ actuales asociados a la posesión de una red duradera de relaciones más o menos institucionalizadas de conocimiento y reconocimiento mutuos" (p. 148). Lo más destacable, es que dichos recursos no son monetarios, y se obtienen por la pertenencia a determinados grupos, relaciones, redes de influencia y colaboración.

El capital social, es fundamental en la gestión de las Relaciones Públicas. INEXMODA ha tejido una red de relaciones profesionales e interpersonales que han favorecido sus acciones. Es este sentido, su Junta Directiva, determinó como prioritario desarrollarlas en beneficio del instituto. Con los viajes internacionales también se aportó a este objetivo. Comenta Alicia Mejía (2016):

La junta directiva muy sabiamente dijo un día, "de Astorga [España] nadie nos va a llamar, nadie va a venir a preguntar qué requerimos. Así que cada uno de ustedes [director de mercadeo y otros], se montan cada uno en un avión y se van a recorrer el mundo para que el mundo los conozca".

Así fue que empezamos a conseguir amigos; a generar una red, yo conocía gente en Barcelona, que después pasaba a trabajar en Madrid, y ese me presentaba otro...nos fuimos volviendo amigos de mucha gente, generando unas cadenas muy bonitas, se nos fueron metiendo sueños locos a la cabeza, porque realmente eran sueños muy locos...

Es así como INEXMODA, empezó a generar vínculos estratégicos a nivel internacional. La siguiente nota del periódico El Colombiano, acerca de la primera edición de Colombiatex, evidencia dicha gestión:

"La misión española viene a Colombia aceptando la invitación realizada por la misión Textil de Colombia, que visitó ese país en octubre de 1988".

El Colombiano, martes 30 de mayo de 1989 , página $6 c$ 
Esa misión, la integraron empresarios y políticos españoles, la lideró Ramón Corbera, director del Instituto Español de la moda.

Uno de los hitos de mayor recordación de las ferias de INEXMODA, fue la participación de Óscar de la Renta en Colombiamoda, en el año 1999. Este diseñador dominicano, perteneciente a la alta esfera de la moda global, realizó un desfile en Medellín, en una época en que la reputación nacional e internacional de la ciudad no era positiva debido a los estragos del narcotráfico.

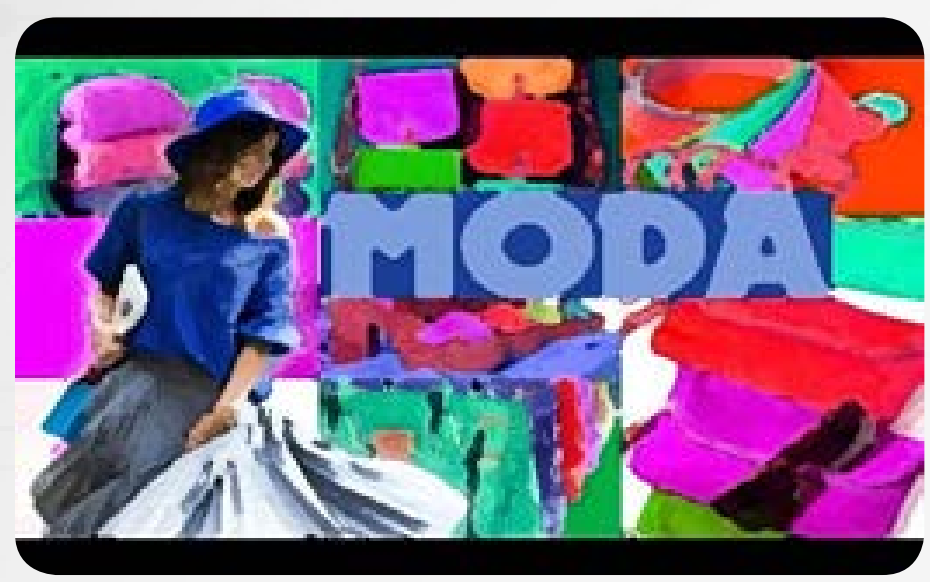

La manera como se logró concretar la presencia de este diseñador en Colombia, es evidencia de una estrategia de Relaciones Públicas, bien ejecutada, así como de la importancia que tiene contar con capital social que acompañe estas estrategias. Comenta Alicia Mejía (2016).

María Emma Mejía [alta funcionaria del Gobierno Nacional y familiar de Alicia Mejía] me ayudó a contactar al diseñador, la señora de Julio Mario Santo Domingo [industrial colombiano], también me colaboró. Hasta que Óscar me llamó un día, y dijo - "Quiero hablar contigo" le dije perfecto. Pero, justo pasó el famoso ataque terrorista a un avión. $Y$ entonces, él me dijo, que no podía venir a Medellín, que tenía compromisos en República Dominicana.

Yo le insistí, le dije, no te preocupes, te mando en avión particular, y salí inmediatamente de la oficina de Óscar, y llamé, de un teléfono público a Carlos Manuel, un empresario amigo mío, me dijo ¿Qué necesitas?- le respondí ¡Tu avioooón!- ¿Para qué?; le expliqué, de inmediato entendió lo que implicaba que Óscar de la Renta fuera a Medellín y me dijo "320... mi piloto se llama... Llámalo y utiliza el avión para lo que tu necesites."

Los empresarios, se ríen mucho, porque dicen que cuando yo los llamaba y les ponía ese tono de voz, ellos decían ¿qué nos va a pedir esta señora?

En la siguiente nota periodística, se muestra comoAlicia Mejía recibió el apoyo de distintas personalidades, en los hechos referentes a la presencia de Óscar de la Renta en Medellín, el acompañamiento lo tuvo, además de personajes de la esfera empresarial, la política y medios de comunicación.

"Alicia Mejía, directora de Colombiamoda, Juan Gómez Martínez, Alcalde de Medellín y José Lavatón, gerente del Hotel Intercontinental, dan la bienvenida al diseñador Óscar de la Renta, a su llegada al aeropuerto."

El Colombiano, jueves 26 de agosto de 1999, plana 2. avión de Carlos Manuel Echavarría) les sirve a todos. En esta perspectiva se debe destacar la gestión del capital social, regido básicamente por relaciones simbólicas y de pertenencia, que el Instituto, desarrolló en beneficio del sector al que servía. Martha Cálad (2016) comenta al respecto:

"INEXMODA, en esencia cuenta con el apoyo de los industriales, entonces cuando se necesita algo, ellos lo hacen con todo el amor, porque saben que es en beneficio del sector."

Luego de lograr el acercamiento y la presentación de Óscar de la Renta en Colombiamoda, la relación entre Alicia Mejía y el diseñador, posibilitó que otros representantes de la moda se aliaran con INEXMODA. Es el caso de Alain Hivelin. Expresa Alicia Mejía (2016):

Óscar me había conseguido una cita con Narciso Rodríguez [diseñador estadounidense] y se dañó, llegué a su oficina, y me dice: Narciso no es, ¿Yo qué voy a hacer? Pero inmediatamente cogió el teléfono y llamó a París al presidente de la casa Balmain, Alain Hivelin, y le dijo, $-i$ Por qué no vamos con la colección de alta costura a Colombia?- Yo miraba a Óscar y ise me salían los ojos!, y Alain le dijo: -ah bueno, vamos a Colombia. -Me prestó la colección de alta costura, ¡alta costura! ¡Latinoamérica nunca había visto eso!

El desfile de Belmain, se llevó a cabo un el 2000, en el 2003, se hizo exhibición retrospectiva de la misma marca en el Museo Nacional en Bogotá, y en el 2004, en el Museo de Antioquia. Todo esto generó notas de prensa en diversos periódicos. El Tiempo, un martes 4 de noviembre de 2003, publicó:

Por primera vez en su historia, el Museo Nacional de Colombia exhibe una muestra dedicada a la moda, Pierre Balmain, arquitecto de la moda. 19452002. Un paso que hasta hace muy poco tiempo dieron instituciones como la Royal Academy of Art, de Londres, que el pasado 18 de octubre abrió una exposición retrospectiva del diseñador italiano Giorgio Armani (1934).

Tengo un sueño, quiero llevar a Colombia, algún día, una retrospectiva de Pierre Balmain , le dijo, en París y a comienzos del 2003, Alicia Mejía, directora de mercadeo de Inexmoda, a Alain Hivelin, dueño de la Casa Balmain. Hivelin, que visitó Colombia en el 2000 junto a Oscar de la Renta, director creativo de esta institución hasta el 2002, abrió los armarios de la Casa Balmain para que Julián Posada, diseñador antioqueño y curador de la muestra; José Ignacio Casas, coordinador del proyecto, y Alberto Sierra, quien hizo el montaje museográfico de la exposición en el Museo de Antioquia, eligieran todo lo necesario para la retrospectiva. (página 2-2).
Bourdieu, indica que el capital social se trata de la "totalidad de recursos basados en la pertenencia a un grupo" (1983, p.148) y que el capital total que poseen los miembros individuales del grupo (en este caso, el 
La Revista Semana, también, publicó acerca de los desfiles:

La presentación de la colección Otoño-Invierno 2000-2001, la casa francesa Balmain será el acontecimiento más importante de moda que se ha visto en el país. Este año se invitó a varios diseñadores de renombre y la noticia grande es que la casa francesa Balmain aceptó la invitación y llega con su última colección de alta costura. La tradicional Casa de Modas, cuyo fundador fue Pierre Balmain y su modisto actual es Oscar de la Renta. Un gran paso para esta pasarela porque por primera vez una casa de alta costura acepta traer su colección más reciente. Gracias a esto se verán sobre la pasarela de Orbitel los 60 trajes que se presentaron el mes pasado en París de la colección Otoño-Invierno 2000-2001 —garantía de que realmente es lo último-.

(11 de septiembre del 2000)

Para Bourdieu, el capital social poseído por un individuo "dependerá tanto de la extensión de la red de conexiones que este pueda efectivamente movilizar, como del volumen de capital poseído por aquellos con quienes está relacionado" (1983, p.150), a su vez, el capital social ejerce un efecto multiplicador del cual los beneficios que se obtienen por la pertenencia a un grupo, componen el "fundamento de la solidaridad que los hace posibles".

En el siguiente diagrama, se presenta la ruta que siguió Alicia Mejía (directora de mercadeo de Inexmoda en Medellín), para lograr contacto con Alain Hivelin, presidente de la casa de modas francesa Balmain. Esta ruta coincide con la teoría "seis grados de separación" propuesta por Duncan Watts (2003), en la que propone que una persona puede conectarse con cualquier otra persona con menos de cinco intermediarios.

Es posible representar el proceso con un diagrama:

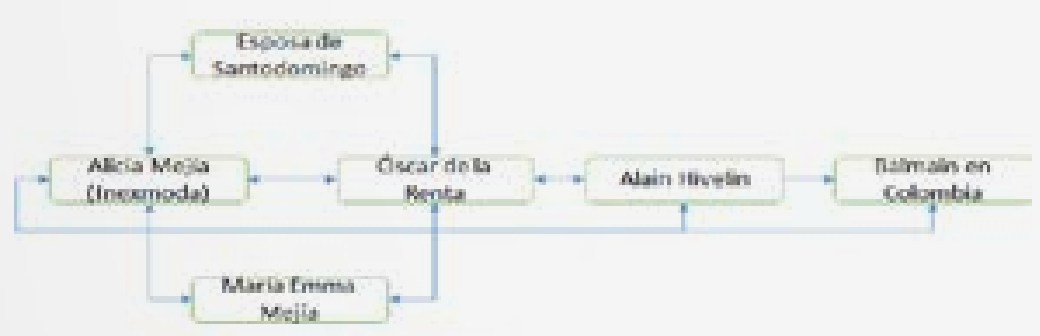

Fuente. Elaboración propia

Un proceso similar ocurrió entre Alicia Mejía (INEXMODA), PROEXPORT (actualmente PROCOLOMBIA), Lina Moreno de Uribe (Primera Dama de Colombia, en la época), Cecilia Duque (Artesanías de Colombia), Fabio Valencia (Embajador de Colombia en Italia) y Mario Bocelli (Presidente de la Cámara Italiana de la Moda), el relacionamiento entre estos, permitió hacer realidad el proyecto Identidad Colombia, en la semana de la moda de Milán, Italia. Al respecto comenta Alicia Mejía (2016).

Lina me llama un día, recién entrado el período presidencial de Álvaro Uribe, me voy para donde ellos, allá en la casa presidencial; y en la cocina, estábamos comiendo arepa, y me dice, "estoy recorriendo el país, estoy conociendo las unidades del país; o los ayudamos a que vivan de su producto, o se tienen que ir a la guerrilla o al narcotráfico. Piensa en un proyecto". Mientras tanto Cecilia Duque de Artesanías, llevaba dos años diciéndome, "moda y artesanía, artesanía y moda... hagamos algo Alicia". Yo salí de la Casa de Nariño y en la calle llamé a Ceci, y le dije "Ceci, ya me llegó la idea..

Identidad Colombia, fue el acontecimiento internacional de mayor escala que Mejía gestionó en INEXMODA, significó la visibilización no solo del instituto, sino del país, en el sector de moda europeo, generando interés de empresarios e internacionalizando al país. En Extroversia, el 8 de octubre de 2005, se publicó:

La mezcla de elementos de tradición indígena y artesanal con tips de modernidad fueron los que brillaron en la pasarela "Identidad Colombia", que se presentó en Milán. Tres diseñadores colombianos mostraron sus diseños con 'Identidad Colombia', una colección $100 \%$ nacional, con la que trataron de desafiar al mundo de la moda en las pasarelas de Milán, Italia. Hernán Zajar, Amelia Toro y Beatriz Camacho, fueron los encargados de clausurar la semana de la moda "pret a porter" de Milán, con un desfile original, étnico y muy colombiano.

El periódico El Tiempo, también reseñó este acontecimiento:

\section{Así llegamos a Milán}

Valencia Cossio, días atrás, le había dicho a la gerente general de Artesanías de Colombia, Cecilia Duque, que la verdadera política estaba en la cultura. Consciente de ello, aprovechó sus argucias para convencer a Boselli de la importancia de que patrocinara el Concurso Internacional de Diseño para la Artesanía durante la semana de la moda en Milán. Boselli aceptó sin refutar.

Se había abierto así la primera puerta. Valencia Cossio, a su regreso a Italia, recibió una carta de Boselli en la que el italiano se deshacía en elogios por aquella pasarela afortunada. Quello che abbiamo visto a Colombiamodaci ha molto favorevolmente colpito (Aquello que vimos en Colombiamoda nos sorprendió muy favorablemente). El entusiasmo era tanto que el embajador, en diciembre, se atrevió a provocarlo con una propuesta: ¿Sería posible -le preguntó- tener la pasarela en la semana de la moda de Milán? La respuesta le cortó la respiración: Es posible, dijo Boselli.

Valencia Cossio corrió a hablar con Cecilia Duque y con la Primera Dama, Lina Moreno, para poner en hombros de la última, la gestión final de abrir las puertas en Europa. Les habló de la carta de Boselli en la que se declaraba feliz por el éxito de Silvia Tcherassi y por la interpretación hecha por varios diseñadores usando productos artesanales. Lina de Uribe aceptó, pero les pidió ayuda.

El siguiente paso era conseguir el dinero. Alicia Mejía, directora de mercadeo de Inexmoda, y Luis Guillermo Plata, presidente de Proexport, se reunieron con Valencia Cossio y escucharon su propuesta. Les pareció una locura. Lo era. Y ambos tenían razón: era prematuro, se necesitaba más tiempo y era una empresa costosa. Valencia Cossio no cedió. Es la oportunidad de la vida, les dijo. Entonces, es ahora o nunca.

Accedieron dudando. Pero pronto se llenaron de ahínco. Proexport pisó el negocio para los nueve días de Milano Moda Donna, con 20 mil euros, y las empresas Orbitel, Bellsouth e Invista aseguraron 10 mil euros cada una. La pasarela era un hecho, y Boselli confirmó que se haría en la clausura. Además, se consiguió un stand para presentar el trabajo del que subsisten 350 mil artesanos en el país.

(25 de enero de 2004) 
La realización de proyectos como Identidad Colombia en Milán, no solo dan cuenta del impacto mediático y de posibilidades empresariales, sino de la integración de distintas instituciones en búsqueda de un objetivo en común, para el caso fueron: Orbitel, Bellsouth, Invista, la Presidencia de Colombia, Artesanías de Colombia e Inexmoda.

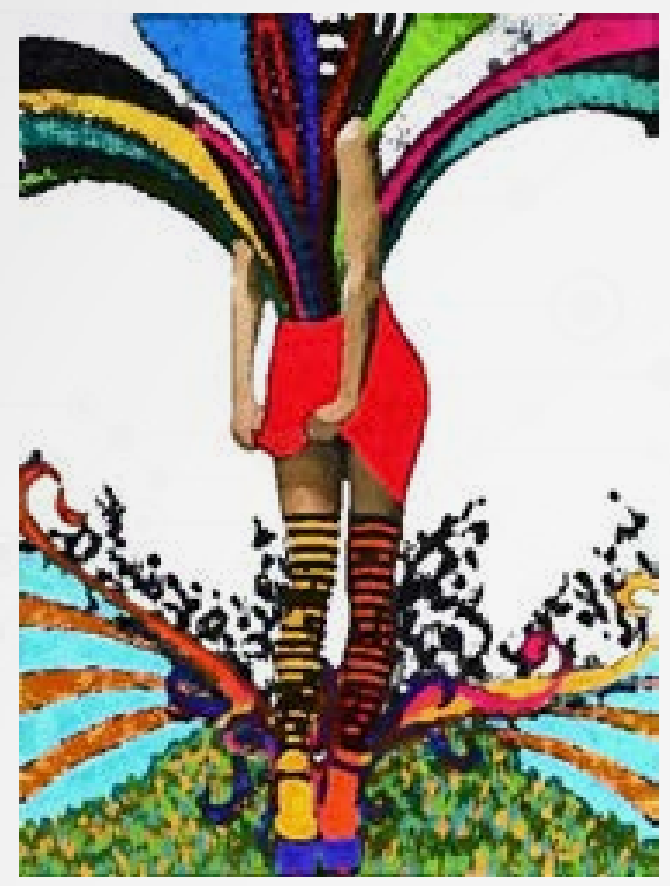

2.3 Aliados: apoyo interinstitucional para la ejecución de estrategias de Relaciones Públicas

Con el capital social encargado de las relaciones interpersonales e incluso profesionales, centralizadas en personajes específicos, se hace necesario abordar la relación con otro de los públicos clave en las estrategias de Relaciones Públicas de la gestión de comunicación de INEXMODA: las instituciones, organizaciones y empresas.

Las relaciones positivas con otras instituciones, fueron una estrategia que favoreció la ejecución de distintos proyectos en INEXMODA. Se destacan instituciones como la Asociación Nacional de Industriales (ANDI), Procolombia (anteriormente Proexpo y Proexport) y Avianca.

La Aerolínea, Avianca, está en la memoria de INEXMODA porque al segundo o tercer año que nos propusimos invitar prensa, nos apoyaron con el traslado de periodistas de El Tiempo, El Espectador, Cromos. Las empresas de Bogotá empezaron a venir a la feria. PROEXPO, nos colaboró, porque empezó a traer compradores internacionales, eso con mucha dificultad, ya en esa época ir a Medellín era como ir a Siria hoy.

Alicia Mejía, 2016

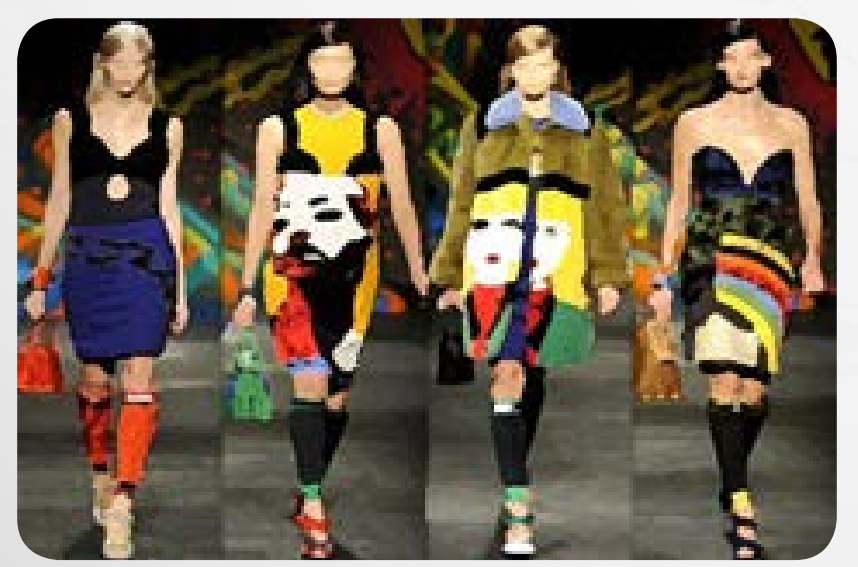

Martha Cálad (2016), también hace referencia al apoyo brindado por Procolombia a INEXMODA.

\begin{abstract}
"PROCOLOMBIA... sin ellos no se habría podido llegar a darle el reconocimiento que tiene la feria a nivel internacional, porque con ellos se ha hecho, todos los años, un trabajo en conjunto para traer compradores internacionales, tanto a COLOMBIATEX como a COLOMBIAMODA."
\end{abstract}

Entendiendo las relaciones públicas como una disciplina "socio-técnico-administrativa" (Wilcox, 2006, p.9), en la que las estrategias son planeadas, se destaca la gestión en INEXMODA, en cuanto sus distintos públicos. Cuando el público de interés resulta ser una organización, este tipo de estrategia es definida por Wilcox como una "relación sectorial". Esto le permitió al instituto, acercarse a diferentes entidades para interactuar con ellas, en busca de un bien mutuo.

Las articulaciones interinstitucionales de INEXMODA, han sido motivo de diversas notas de prensa, el interés de estas, la moda como uno de los potenciadores de la economía nacional. Diversos tipos de alianzas se han realizado en el instituto, no solo en la ejecución de proyectos, sino de estrategias en distintos ámbitos. El Mundo, destacó el 3 de julio de 2008:

\begin{abstract}
Avianca, por ejemplo, suscribió un convenio con Inexmoda, entidad organizadora de Colombiamoda y la Asociación Hotelera de Colombia, Cotelco, para que quienes se desplazan desde otras ciudades hacia Medellín, puedan disfrutar de algunos descuentos. El convenio, ofrecido a expositores, visitantes y demás interesados, les permitirá disfrutar de beneficios tarifarios en los tiquetes aéreos, alojamiento, desayunos, traslados desde el aeropuerto y al recinto ferial, además de seguro hotelero.
\end{abstract}

\subsection{Legitimidad: validadores}

Para Wilcox, en las relaciones públicas se debe "segmentar cuidadosamente el público en función de diversas características demográficas y psicológicas," (2006, p. 20). Las estrategias de Mejía en INEXMODA, podrían ser clasificadas de acuerdo a su función. En este caso, estrategias de posicionamiento (para ganar un lugar en las ferias de moda del continente), y de publicity (para obtener notoriedad en Colombia).

Los validadores, son personajes o instituciones con la capacidad de suplir los dos estrategias, se puede inferir que los validadores principales durante la gestión de Mejía, en INEXMODA, fueron los periodistas y diseñadores internacionales que ratificaran la calidad de los proyectos. Julián Posada (2016), comentó al respecto:

Un diseñador internacional, lo poníamos a mostrar su colección en un local, para darle la oportunidad a este último, de fogueo, esto es que lo viera la prensa y que pudiera mostrarse ante compradores.

A partir de la revisión de prensa, se presenta el siguiente cuadro en el cual se ubica a los validadores del proyecto INEXMODA (ver tabla N2). 
Tabla N2.

Validadores de INEXMODA

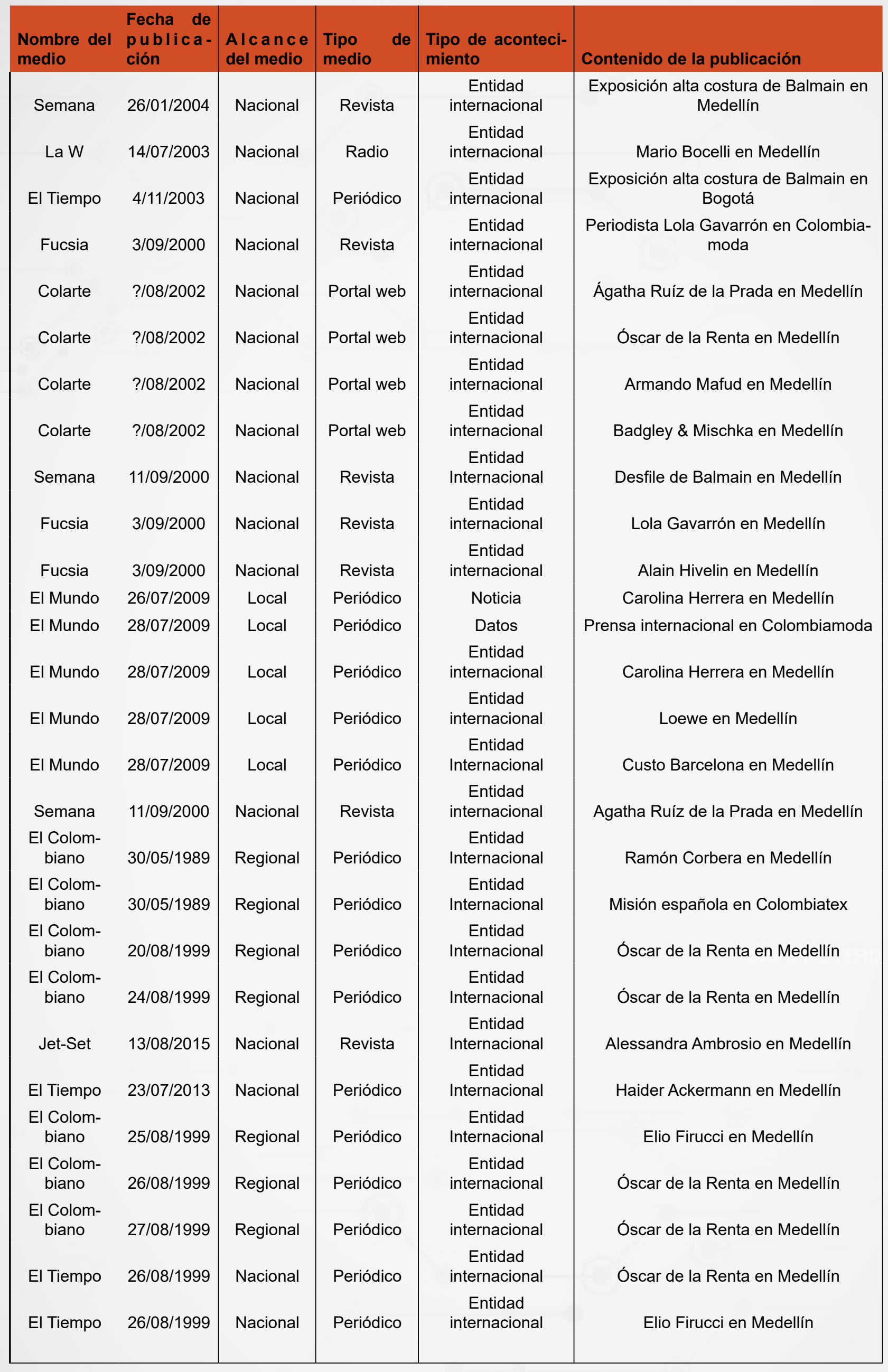

Fuente. Elaboración propia 
Eso responde a una nueva estrategia, y es darle fuerza al talento colombiano. Lo hemos hecho a través de diferentes maneras, con el concurso de "Latinoamérica habla" dirigido a los estudiantes de cualquier disciplina al diseño; se convoca a todas las universidades de Latinoamérica. [Otra estrategia ha sido la inclusión]... Volver nuestras ferias absolutamente incluyentes, que Colombiamoda brinde el espacio para empresas que están dirigidas al mercado masivo, tengan presencia en la feria... Ellos empezaron con su feria aparte, y lo hicieron por varios años, pero INEXMODA se les acercó y les dijo "Vengan, hagan parte de nuestra feria" y por eso hemos tenido, durante varios años, a Moda para el Mundo dentro de Colombiamoda.

Martha Cálad, 2016

En el caso de la gestión de la comunicación de INEXMODA, es posible afirmar que las ferias Colombiamoda y Colombiatex, en tanto producto del Instituto, comunican su procedencia. En la gestión de Alicia Mejía las estrategias de Relaciones Públicas, atrajeron validadores de talla internacional como: Óscar de la Renta, Balmain, Badgley Mischka, Carolina Herrera, Loewe, Ágatha Ruíz de la Prada, entre otros.

2.6 Impacto de la gestión de la comunicación y las estrategias de relaciones públicas en Inexmoda: el publicity en la consolidación de Medellín como ciudad de moda.

Para Annie Bartoli en Comunicación y organización: la organización comunicante y la comunicación organizada (1992), la información externa de notoriedad consiste en que la organización informa con el fin de dar a conocer sus productos o mejorar su imagen. Las principales formas de información externa es la publicidad, la promoción, las donaciones, los patrocinios, las intervenciones en manifestaciones o coloquios, artículos sobre la empresa publicados en distintos medios, las acciones de difusión, las demostraciones de productos y visitas a la empresa, la presencia en las instituciones educativas, y de stands de la empresa en ferias y exposiciones.

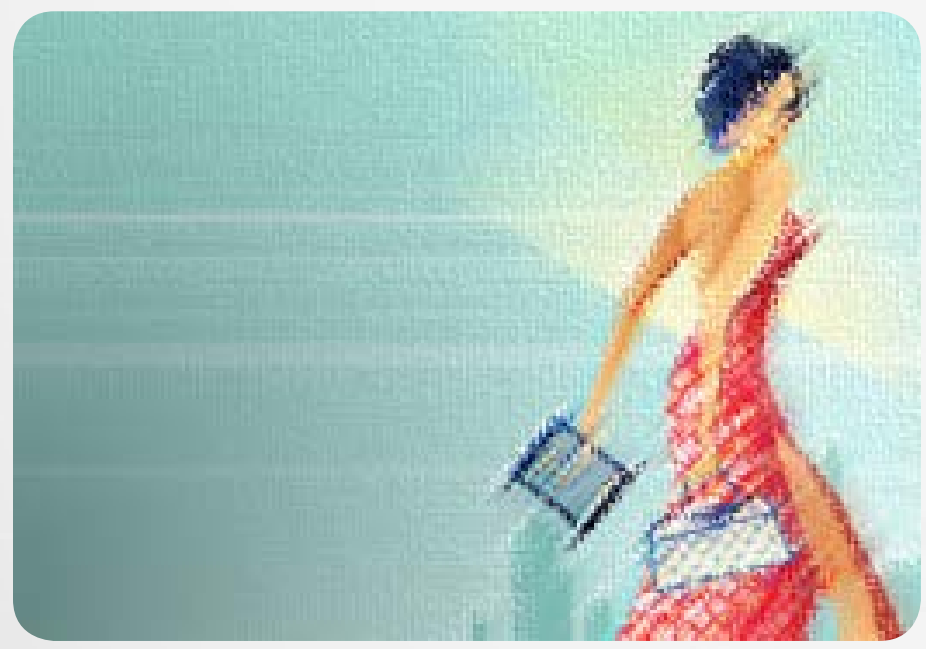

Siendo el publicity la información que un medio de comunicación difunde sobre actividades, actos o acontecimientos de una organización, mediante noticias que no subscribe la organización sino el medio de comunicación, es importante resaltar que es la organización "quien controla el mensaje, y que suele ser el resultado informativo de una acción de comunicación" (Wilcox, 2006, p.13), estratégicamente planeada.
La realización de Colombiatex y Colombiamoda, ejecutados porAlicia Mejía en INEXMODA, conllevaron publicity, de igual manera, los eventos programados fuera del escenario tradicional de las ferias, tales como Identidad Colombia en Milán o la exhibición de Balmain en los museos de Bogotá y Medellín, que lograron generar atención mediática en función a los intereses de la organización, en fechas y escenarios distintos, a los de las dos ferias más importantes de la organización.

Para la investigación, como ya se dijo, las estrategias de relaciones públicas se midieron de acuerdo al concepto de equivalencia publicitaria "aparecer en un medio debido a un hecho noticioso, no una pauta" (Wilcox, 2006, p. 207)

\section{Conclusiones}

La constitución de Medellín como ciudad de moda en Colombia y Latinoamérica, se presenta a través de estrategias como el benchmarking, mediante el cual se adaptaron referentes europeos a las necesidades locales, permitiendo el surgimiento de ferias como Colombiatex y Colombiamoda: Capital social, con el que se generaron relaciones positivas para INEXMODA y ejecutar estrategias de alto impacto con validadores y personalidades internacionales, apoyo interinstitucional, con diversas empresas que han trabajado en conjunto con el instituto. Y la gestión del publicity, para que los medios dieran a conocer los resultados de las estrategias a nivel nacional e internacional.

Entre los años 1989 y 1990, en la gestión deAlicia Mejía, se implementó el benchmarking, se constituyeron y consolidaron los dos productos principales del Instituto para la Exportación y la Moda: Colombiamoda y Colombiatex. Ello con el apoyo de organizaciones como Proexpo (actualmente Procolombia). Entre los años 1999 y 2006, estando las ferias consolidadas, las estrategias se enfocaron a ratificar la calidad de los productos de INEXMODA, por medio de distintos validadores y la publicity.

En la actualidad, las estrategias parecen seguir el mismo modelo que las ejecutadas en el periodo de Mejía, y se les ha adicionado la perspectiva de moda nacional. Se destaca también el componente académico, que se ha incorporado.

La equivalencia publicitaria (aparecer en un medio debido a un hecho noticioso, no una pauta) es un concepto que se retoma del manual de Wilcox, "Relaciones Públicas: estrategias y tácticas" (2006) para analizar la publicity, en INEXMODA, se observó que esta ha impactado positivamente en el posicionamiento de Medellín como ciudad de moda. Se encontró que año tras año, desde 1989, se publica gran cantidad de contenido respecto al Instituto y sus ferias en relación con la ciudad de Medellín. A partir de 1999, la gestión del publicity se toma los medios nacionales e internacionales. En los últimos años, por medio de la web, el contenido de lo que ocurre en las ferias es compartido vertiginosamente en internet [2]. 


\section{Bibliografía}

- Sautu, R. "Eldiseño de una investigación: teoría, objetivos y métodos”. En: Todo es Teoría. Buenos Aires, Argentina. 2005, p 21-41. Recuperado de: https://drive.google.com/file/ d/OB2xFycSbB3m4NHIBSHdjODI2UVk/view

- Martínez Sánchez, A. (1994). "El estudio de casos en los procesos de intervención social". En: El estudio de casos como estrategia didáctica para la formación de los profesionales en la acción social. Madrid: Narcea.

- Wilcox, D. (2006). "Relaciones públicas: estrategias y tácticas". Madrid: Pearson Educación.

- Goetsch David L.. (2003, marzo 6). Teoría del Benchmarking. Recuperado de http://www. gestiopolis.com/teoria-del-benchmarking/

- Agudelo, V. (2015). Las ferias como escenarios de comunicación y promoción de la ciudad (caso Inexmoda). Trabajo de grado. Universidad de Antioquia.

- Gartner, P. (2012) El vestir como referente de transformaciones sociales en Medellín: un análisis de la industrialización textil y del surgimiento de Inexmoda como estrategias de modernización y cambio social. Trabajo de grado. Universidad de Antioquia.

- Bürdek, B. (2002). Diseño. Historia, teoría y práctica del diseño industrial. Editorial Gustavo Gili: Barcelona. (pp. 279-295)

- Bartoli,A. (1992)Comunicación y Organización. Edt. Paidós Empresa. Barcelona. 1992.

- Gorbis, Marina. Traducida por Castillón, Juan Carlos. (2012). "Capital Social”. En Revista El Malpensante, edición No. 127. Recuperado de http://www.elmalpensante.com/articulo/2360/ capital_social

- Bourdieu, Pierre (1983). Poder, Derecho y Clases Sociales. Desclée. pp. 131-164.

- Mejía, M.A. (2016) Entrevistada por Roncancio, P. Medellín.

- Cálad, M. (2016) Entrevistada por Roncancio, P. Medellín.

- Posada, J. (2016) Entrevistado por Roncancio, P. Medellín.

- Ospina, R. Entrevistado por Inexmoda. Medellín.

- Semana. Edición 1134. (2004) "Alta costura" Recuperado de: http://www.semana.com/ sociedad/articulo/alta-costura/63063-3

- La W. (2003) Entrevistas W "Fabio Valencia Cossio, embajador en Italia; Mario Bocelli, presidente de la Cámara Nacional de la Moda Italiana y Alicia Mejía". Recuperado de: http://www.wradio.com.co/escucha/ archivo_de_audio/fabio-valencia-cossioembajador-en-italia-mario-bocelli-presidentede-la-camara-nacional-de-la-moda-italiana-yalicia-mejia/20030714/oir/691.aspx
- El Tiempo. (2003) “Un arquitecto de la moda” en archivo. Recuperado de: http://www.eltiempo. com/archivo/documento/MAM-1005039

- Fucsia. No. 03 de septiembre. (2000) "La magia de Silvia Tcherassi". Recuperado de: http://www.colarte.com/colarte/ConsPintores. herassiSilvia $\% 24 \% 241$ col\%24\%24Fucsia900. html

- Colarte. (2002) "Colombiamoda, última moda en Medellín”. Recuperado de: http:// www.colarte.com/colarte/ConsPintores. General\%24\%241col\%24\%24Colombiamoda. html

- Semana. (2006) "La vitrina vista año tras año" Especiales. Recuperado de: http://www. semana.com/especiales/articulo/la-vitrinavista-ano-tras-ano/79832-3

- El Colombiano Blogs. (2000) “En 1990 cuando empezó Colombiamoda, Medellín no tenía metro". Recuperado de: http://www. elcolombiano.com/blogs/elcoleccionista/ colombiamoda-empezo-en-1990-el-ano-de-laaepertura-economica/52

- Semana. (2000) "Alta costura" Vida moderna. Recuperado de: http://www.semana.com/vidamoderna/articulo/alta-costura/43272-3

- El Tiempo. (2010) "5 momentos clave de Colombiamoda". Archivo. Recuperado de: http://www.eltiempo.com/archivo/documento/ MAM-4067321

- El Mundo. (2009) "Los 20 de Colombiamoda". Recuperado de: http://www.elmundo.com/ portal/resultados/detalles/?idx=122937\# . WAnDoeDhAdV

- El Mundo. (2009) "20 años posicionando moda". Recuperado de: http://www.elmundo. com/portal/pagina.general.impresion. php?idx=123150

- La Hoja de Medellín. No.43, junio. (1996) "Alicia Mejía".

- El Colombiano. Mayo 29, (1989)

- El Colombiano. Mayo 30, (1989)

- El Colombiano. Agosto 20, (1999)

- El Colombiano. Agosto 24, (1999)

- El Colombiano. Agosto 25, (1999)

- El Colombiano. Agosto 26, (1999)

- El Colombiano. Agosto 27, (1999)

- El Tiempo. Agosto 24, (1999)

- El Tiempo. Agosto 25, (1999)

- El Tiempo. Agosto 26, (1999)

- El Espectador. Agosto 25, (1999) 
Notas

[1] María Alicia Mejía (Medellín, 1946), nieta del industrial antioqueño Gonzalo Mejía Trujillo, fue la directora de mercadeo de Inexmoda desde 1988 hasta el 2006. En dicho cargo creó y dirigió Colombiatex en 1989 y Colombiamoda en 1990.

[2] El autor brinda el agradecimiento al Fondo para Apoyar los Trabajos de Grado de Pregrado de la Facultad de Comunicaciones, y al Comité para el Desarrollo de la Investigación de la Universidad de Antioquia, que brindaron su apoyo en el desarrollo de la investigación.

\section{Para citar este artículo:}

Roncancio, Pablo (2017). Relaciones Públicas. Estrategias implementadas por el Instituto para la exportación y la moda - Inexmoda.

Revista Luciérnaga / Comunicación , Año 9, N17.

Facultad de Comunicación Audiovisual- Politécnico

Colombiano Jaime Isaza Cadavid- PCJIC \& Facultad

de Ciencias de la Comunicación - Universidad

Autónoma de San Luis Potosí- UASLP. México.

$$
\text { Págs. 24-37. }
$$

DOI. 10.33571/revistaluciernaga.v9n17a4 orcid.org/0000-0002-8012-0568

OJS. http://revistas.elpoli.edu.co/index.php/luc/issue/ archive

Link. http://www.politecnicojic.edu.co/index.php/ revista-luciernaga

\section{Anexo 1.}

\section{Parrilla de desfiles Colombiamoda 2013.}

\section{•Haider Ackermann.*}

American Express de Bancolombia by Francesca Miranda.

- Fucsia - Haceb by Manuela Álvarez.

- Alcaldía de Medellín by Especia, Mon \& Velarde y Whisper \& Loud.

- Ipanema by Paradizia.

- Studio F.

- Pilsen + Coltejer by Daniel Hoyos.

- Arkitect by Pink Filosofy.

- Carrusel by Adriana Santacruz.

- Pasarela Tennis.

- Babalú.

- Infashion - Blancox: 5 talentos.

- Fundación Universitaria del Área Andina.

- Jóvenes Creadores Chocolyne - Colegiatura.

- Kotex by Agua Bendita.
- Vélez.

- Cromos - Oral B by Polite.

- Leonisa.

- Camilo Álvarez.

- Isabel Henáo.

- Colombia Diseña by Falabella: Maria E. Villamil, Duplicity, Daniel Hoyos, Carolina Sepúlveda, A New Cross y Warmi.

- Rapsodia.

- UPB.

- El Colombiano by Maaji.

- Carolina Sepúlveda.

- Pasarela Dorada Pantene by Renata Lozano.

- Argos by Silvia Tcherassi.

\section{Parrilla de desfiles Colombiamoda 2014:}

Non Stop: Faride Ramos, Julián Ruiz, Manuela Álvarez, 747

- Non Stop: Bastardo, Nilos, Maria Angélica Guerra, Andrés Pajón

- Trucco's

- Non Stop: Mariana Vélez, Purpuratta, Alado, Lia Samanta

- Fucsia - Ashes

- Infashion - Kika Vargas

- Beverly Hills Beachwear

- Fundación Universitaria del Área Andina

- Appicaps y Seletiva Calzado - Vestuario de Portugal

- Diseñadores de Cúcuta

- Pasarela UPB

- Alcaldía de Medellin: Andrea Landa, Natalia Londoño y Love Citizens

- Jóvenes Creadores Chocolyne - Colegiatura

- Modo RosaColectivo de diseñadores

- Paradizia Beachwear

- Agua Bendita

- Onda de Mar

- Agua Cielo - Don Eloy by Carolina Sepúlveda

- Studio F

- Matisses by Jorge Duque

- Leonisa

- Cromos - Renata Lozano

- Punto Blanco

- Haceb - Custo Barcelona

- Carrusel - Banco de Bogotá-Beatriz Camacho 
- Lina Cantillo

- Pasarela Argos by Camilo Álvarez

- Tennis

- GEF

- Johanna Ortiz

\section{Parrilla de desfiles Colombiamoda 2015:}

Non Stop Moda a la Colombiana Andrea Landa,Daniela Battle, Natalia Londoño

- Plaza Mayor 40 años / Tous by Jorge Duque

- Fundación Universitaria del Área Andina

- Alcaldía de Medellín by Andrés Pajón

- Aló by Luli Fama

- Falabella

- Carrusel y Banco de Bogotá by Leal Daccarett

- Vidrio es vida by Pepa Pombo

- Leonisa

- Non Stop Moda la Colombiana Erikó by David Alfonso, Orozco Clothing, Mon \& Velarde

- Trucco's Jeans

- Infashion / Lycra by Isabel Caviedes

- Apiccaps - Calzado de Portugal

- Jóvenes Creadores Chocolyne - Colegiatura

- Paradizia

- Studio F

- New Balance by DaríoCárdenas

- Dafiti by Johanna Ortiz

- Non Stop Moda a la Colombiana Mar de Rosas, French Vanilla, Love Citizens

- Fucsia 15 años - Banco de Bogotá by Carlo Carrizosa y Papel de Punto

- M Francesca Miranda, Argemiro Sierra, Juan Pablo Socarrás, Manuela Álvarez, Ana María Restrepo

- UPB

- Beverly Hills

- Ricardo Pava

- Arkitect: Isabel Henao, Renata Lozano, Jorge Duque, Camilo Álvarez

- Punto Blanco

- Glade by Kika Vargas
Parrilla de desfiles Colombiamoda 2016:

- Pasarela inaugural: Ron Medellín presenta a Jorge Duque

- Non Stop 1 Moda a la Colombiana: Mulierr, Sort Lingerie

- Pasarela Origen-USAID Edwing D’Angelo

- Fundación Universitaria del Área Andina

- Pasarela Alcaldía de Medellín Épica by Orozco Clothing

- Carmen Steffens

- Leonisa 60 años

- Cromos 100 años Pepa Pombo by Mónica Holguín

- Non Stop 2 Moda a la Colombiana: Carlo Carrizosa, Whitman

- Trucco's Jeans

- Infashion by Andrea Landa

- Premiación concurso Latinoamérica Habla

- Apiccaps - Asociación Portuguesa de la Industria del Calzado

- Jóvenes Creadores - Colegiatura

- Inizio

- New Balance + Pilatos

- Carrusel - Banco de Bogotá by Andrés Pajón

- Jon Sonen Johanna Ortiz Non Stop 3 Moda a la Colombiana: Entreaguas, Noise Lab

- Fucsia - Finesse - Concurso "Se busca diseñador" Francris Arata Bastardo de Rubén Antorveza

- Pasarela M: Pura, Noise Lab, Airavata

- UPB - Universidad Pontificia Bolivariana

- Pasarela Origen- USAID- Lía Samantha

- Ann Chery

- Bronzini

- Mr. Tea presenta Agua Bendita

- Erikó by David Alfonso

- Neutrogena - Descubre que es posible by Renata Lozano

*Las pasarelas de diseñadores y marcas en negrita son internacionales. 